\section{(2) OPEN ACCESS}

\title{
Gender differences in clinical presentation and 1-year outcomes in atrial fibrillation
}

\author{
Renate B Schnabel, ${ }^{1}$ Ladislav Pecen, ${ }^{2}$ Francisco M Ojeda, ${ }^{1}$ Markus Lucerna, ${ }^{3}$ \\ Nargiz Rzayeva, ${ }^{1}$ Stefan Blankenberg, ${ }^{1}$ Harald Darius, ${ }^{4}$ Dipak Kotecha, ${ }^{5}$ \\ Raffaele De Caterina, ${ }^{6,7}$ Paulus Kirchhof ${ }^{8,9}$
}

\begin{abstract}
- Additional material is published online only. To view please visit the journal online (http://dx.doi.org/10.1136/ heartjnl-2016-310406).
\end{abstract}

For numbered affiliations see end of article.

Correspondence to Dr Renate B Schnabel, University Heart Center, Department of General and Interventional Cardiology, Martinistr. 52, 20246 Hamburg, Germany; r.schnabel@uke.de

Received 2 August 2016 Revised 14 December 2016 Accepted 15 December 2016 Published Online First 22 February 2017

\section{ABSTRACT \\ Objectives Our objective was to examine gender differences in clinical presentation, management and prognosis of atrial fibrillation (AF) in a contemporary cohort.}

Methods In 6412 patients, $39.7 \%$ women, of the PREvention of thromboembolic events - European Registry in Atrial Fibrillation, we examined gender differences in symptoms, risk factors, therapies and 1-year incidence of adverse outcomes.

Results Men with AF were on average younger than women (mean \pm SD: $70.1 \pm 10.7$ vs $74.1 \pm 9.7$ years, $\mathrm{p}<0.0001$ ). Women more frequently had at least one AF-related symptom at least occasionally compared with men (95.4\% in women, $89.8 \%$ in men, $\mathrm{p}<0.0001$ ).

Prescription of oral anticoagulation was similar, with an increase of non-vitamin $\mathrm{K}$ antagonist oral anticoagulants from $5.9 \%$ to $12.6 \%$ in women and from $6.2 \%$ to $12.6 \%$ in men, $p<0.0001$ for both. Men were more frequently treated with electrical cardioversion and ablation (20.6\% and $6.3 \%$, respectively) than women (14.9\% and $3.3 \%$, respectively), $p<0.0001$. Women had $65 \%$ (OR: $0.35 ; 95 \% \mathrm{Cl}(0.22$ to 0.56$)$ ) lower age-adjusted and country-adjusted odds of coronary revascularisation, $40 \%$ (OR: 0.60; (0.38 to 0.93$)$ ) lower odds of acute coronary syndrome and 20\% (OR: 0.80; (0.68 to 0.96)) lower odds of heart failure at 1 year. There were no statistically significant gender differences in 1-year stroke/transient ischaemic attack/arterial thromboembolism and major bleeding events.

Conclusion In a 'real-world' European AF registry, women were more symptomatic but less likely to receive invasive rhythm control therapy such as electrical cardioversion or ablation. Further study is needed to confirm that these differences do not disadvantage women with AF.

\section{INTRODUCTION}

Linked

- http://dx.doi.org/10.1136/ heartjnl-2016-311085

CrossMark

To cite: Schnabel RB, Pecen L, Ojeda FM, et al. Heart 2017;103:1024-1030.
Atrial fibrillation (AF) is one of the most frequent cardiovascular diseases and a common comorbidity in older adults in both men and women. ${ }^{1}$ Gender differences in AF risk factor distribution, comorbidities, clinical presentation and cardiovascular outcomes in individuals with $\mathrm{AF}$ have been discussed in the literature in different settings and with conflicting results. ${ }^{23}$ In prior studies, women with AF carried a higher risk of adverse events such as stroke. ${ }^{2}$ The higher risk in women is taken into account in current clinical risk algorithms such as the $\mathrm{CHA}_{2} \mathrm{DS}_{2}-\mathrm{VASc}$ (congestive heart failure, hypertension, age $\geq 75$ years, diabetes mellitus, stroke, vascular disease, age 65-74 years, sex category) score. ${ }^{3}$ Additionally, women appear to have a lower bleeding risk than men. ${ }^{4}$ Less evidence is available on the impact of gender on the complex interaction between $\mathrm{AF}$ and heart failure ${ }^{5}$ and acute coronary syndromes, ${ }^{6}$ which have been increasingly recognised as serious sequelae of AF. In addition, gender-specific clinical predictors of maintenance of sinus rhythm or adequate rate control are sparsely investigated.

Some data suggest gender differences in treatment and response to interventions. ${ }^{45}$ Over the last years, many changes have been witnessed, including an increased prevalence of AF, the introduction of novel therapies and the release of new guidelines.? In particular, non-vitamin $\mathrm{K}$ antagonist oral anticoagulants (NOACs) are increasingly used for stroke prevention in $\mathrm{AF}^{6}{ }^{6}$ Limited data exist on the impact of gender on prescription and continuation of NOACs since the implementation of new AF guidelines. Guidelines recommend that physicians should offer effective diagnostic tools and therapeutic management to women and men equally.

The PREvention of thromboembolic events European Registry (PREFER) in AF is a prospective, observational cohort ${ }^{8}$ implemented to describe the management of patients with AF in Europe after the release of the 2010 European Society of Cardiology Guidelines for the Management of Atrial Fibrillation. ${ }^{9}$ In this study, we focus on gender differences in baseline risk factors, disease history and $\mathrm{AF}$ symptoms, together with therapeutic approaches and 1-year incidence of major outcomes. Our aim is to highlight potential treatment discrepancies in women and men that could impact the prognosis of patients with AF.

\section{METHODS}

\section{Study sample}

Between January 2012 and 2013 the PREFER in AF registry enrolled 7243 patients aged 18 years and older with physician-verified AF across seven Western European countries (France, Germany, Austria, Switzerland, Italy, Spain and the UK). ${ }^{8}$ Eighty-nine per cent of the study participants were treated by cardiologists and recruited from both physician offices and hospitals. One-year follow-up data were obtained from 6412 patients by physician-administered questionnaire and medical chart information. The last follow-up was performed 
in January 2014. Information on individuals without valid follow-up information is provided in online supplementary table 1.

\section{Clinical evaluations}

$\mathrm{AF}$ had to be present on an ECG or in the readout of an implanted device (pacemaker/defibrillator) within the preceding 12 months. Demographic data, clinical variables, disease history and treatment were ascertained by the treating physician. Symptoms related to AF were assessed using the European Heart Rhythm Association (EHRA) score ${ }^{10}$ and included symptoms such as palpitations, fatigue, dizziness, dyspnoea, chest pain and anxiety. The scoring ranged from never, occasional (less than once per month), intermediate (once per month to almost daily) to frequent (at least daily). An individual's EHRA score was assessed by the treating physician as the maximum score of any of the six individual symptom categories. We also assessed the EHRA score in individuals with new-onset AF, which was defined as AF diagnosed fewer than 90 days prior to enrolment. Lack of guideline compliance indicates lack of treatment with oral anticoagulant in the previous 12 months despite guideline indication without contraindication. Adequate heart rate control was defined as a heart rate between 60 and $100 \mathrm{bpm}$ during the clinic visit. The risk of thromboembolic and bleeding events in patients with $\mathrm{AF}$ was assessed by calculating the $\mathrm{CHA}_{2} \mathrm{DS}_{2}$ VASc score and HAS-BLED (hypertension, abnormal renal/liver function, stroke, bleeding history or predisposition, labile international normalised ratio, elderly, drugs/alcohol concomitantly) score. $^{11}$

\section{Statistical analyses}

Data analysis was performed as a substudy of the PREFER in AF registry on the 6412 patients with follow-up information. Complete case analysis was performed, and missing data were assumed to be missing at random. The proportion of women and men lost to follow-up was compared by Fisher's exact test. Variables are presented as number (percentage) or mean $( \pm S D)$, as appropriate. Differences by gender across EHRA score categories for each EHRA symptom and across type of anticoagulation therapy were calculated using Freeman-Halton's extension Fisher's exact test. Gender differences in dichotomous baseline of characteristics and the 1-year incidence of four major medical sequelae of $\mathrm{AF}$ (ischaemic stroke/transient ischaemic attack (TIA)/arterial thromboembolic events, acute coronary syndrome (myocardial infarction and unstable angina pectoris), coronary revascularisation, heart failure and major bleeding events) were examined by multiple logistic regression adjusted for age and country.

\section{One-year predictive models}

An initial round of variable selection was performed, where highly collinear variables and variables with a high number of missing values and/or few events were removed. Subsequently, predictor variables for each logistic regression model were selected by stepwise regression ( $\mathrm{p}=0.20$ for selection and $\mathrm{p}<0.05$ to remain in the model). The list of included predictor variables can be found in online supplementary table 2. Age, gender and country were forced into the regression models. For the outcome heart failure (chronic heart insufficiency and reduced left ventricular ejection fraction), patients with the condition at baseline were excluded from the analysis. Effect estimates for each model are presented separately for women and men. The predictive ability of the derived regressions models compared with the $\mathrm{CHA}_{2} \mathrm{DS}_{2}-\mathrm{VASc}$ and HAS-BLED scores for each outcome was assessed by calculating the area under the receiver operating characteristic curve (AUC), with overoptimism corrected by repeated split sample validation (logistic regression and then AUC computation in randomly split halves of the sample, repeated 100 times).

Analyses were conducted using SAS software V.9.4 (Cary, North Carolina, USA), with a two-tailed significance value of 0.05. Graphics were produced with R V.3.3.0 using the ggplot2 package. ${ }^{12} 13$

\section{RESULTS}

\section{Baseline patient characteristics}

Forty per cent of study participants were women, who were on average 4 years older than men $(74.1 \pm 9.7$ years vs $70.1 \pm 10.7$ years, respectively). The body mass index was comparable in both genders (28.2 $\pm 4.7 \mathrm{SD}$ in men vs $27.6 \pm 5.4 \mathrm{SD}$ in women), and the systolic blood pressure was lower in men than in women $(130.5 \pm 16.1 \mathrm{SD}$ in men vs $132.7 \pm 17.4 \mathrm{SD}$ in women). There were numerous differences in the burden of cardiovascular risk factors and comorbidities according to gender (table 1). Hyperthyroidism, valvular heart disease and antiarrhythmic drug intake were more prevalent in women than in men. Most other cardiovascular risk factors and prevalent disease were more often observed in men. Adequate heart control was comparable in women and men. There was no statistically significant gender difference in the proportion of individuals lost to follow-up, $\mathrm{p}=0.27$.

\section{Atrial fibrillation symptoms}

In men, $90 \%$ experienced symptoms, compared with $95 \%$ in women. Male study participants reported less severe symptoms and showed a lower frequency of symptoms compared with women (figure 1). In both genders, the most common symptoms were fatigue, dyspnoea and palpitations. At least occasional fatigue was reported by $70 \%$ of men and $80 \%$ of women. The least common symptom was chest pain (28\% in men and $32 \%$ in women). The proportions of symptom distribution did not change markedly over the follow-up period (data not shown). In patients with new-onset $\mathrm{AF}$, symptoms were also distributed similarly with women having consistently more frequent symptoms (see online supplementary table 3 ).

\section{Anticoagulation therapies}

Anticoagulation therapy was prescribed in $94 \%$ of both women and men. The majority of patients $(>60 \%)$ were prescribed vitamin $\mathrm{K}$ antagonists at both baseline and 1-year follow-up (figure 2). At both timepoints, more women than men were prescribed vitamin $\mathrm{K}$ antagonists only (baseline: $70 \%$ vs $65 \%$; follow-up: $63 \%$ vs $61 \%$ ), and conversely, more men than women were on a combined vitamin $\mathrm{K}$ antagonist and antiplatelet therapy regimen (baseline: $12 \%$ vs $7.0 \%$; follow-up: $7 \%$ vs $4 \%$ ). A twofold increase in the use of NOACs was demonstrated in both women and men, from about $6 \%$ at baseline to $13 \%$ at the 1-year follow-up.

\section{Therapies to restore sinus rhythm}

Pharmacological and electrical cardioversion attempts were the most common interventions to restore sinus rhythm (table 2). At baseline, women were more likely to have undergone a pharmacological cardioversion attempt compared with men (OR $1.24 ; 95 \%$ CI 1.08 to 1.41$)$. Men had more frequently received electrical cardioversion, catheter ablation (pulmonary vein isolation) or surgical ablation therapy compared with women. The 


\begin{tabular}{|c|c|c|c|c|}
\hline Variables & Women $(n=2546)$ & Men $(n=3866)$ & Women-to-men OR* & $95 \% \mathrm{Cl}$ \\
\hline \multicolumn{5}{|l|}{ Risk factors } \\
\hline Age, years (SD) & $74.1(9.7)$ & $70.1(10.7)$ & & \\
\hline Body mass index, $\mathrm{kg} / \mathrm{m}^{2}$ (SD) & $27.6(5.4)$ & $28.2(4.7)$ & & \\
\hline Systolic blood pressure, mm Hg (SD) & $132.7(17.4)$ & $130.5(16.1)$ & & \\
\hline Ever smoking, n (\%) & $452(18.2)$ & $1958(51.9)$ & 0.21 & (0.19 to 0.24$)$ \\
\hline Alcohol excess (=8 units/week), n (\%) & $14(0.6)$ & $149(3.9)$ & 0.16 & (0.09 to 0.27$)$ \\
\hline Lack of guideline compliance in anticoagulant therapy, $\mathrm{n}(\%)$ & $79(3.1)$ & $83(2.2)$ & 1.17 & (0.85 to 1.62$)$ \\
\hline EHRA score >2, n (\%) & $1564(62.1)$ & 1899 (49.6) & 1.68 & (1.51 to 1.87$)$ \\
\hline $\mathrm{CHA}_{2} \mathrm{DS}_{2}-\mathrm{VASC} \geq 2+, \mathrm{n}(\%)$ & $2235(95.2)$ & $2823(78.9)$ & 5.55 & (4.26 to 7.22$)$ \\
\hline HAS-BLED $\geq 2, n(\%)$ & $1483(70.8)$ & $2072(65.4)$ & 0.85 & (0.74 to 0.97$)$ \\
\hline \multicolumn{5}{|l|}{ Disease history } \\
\hline Diabetes mellitus, $\mathrm{n}(\%)$ & $529(21.0)$ & $893(23.3)$ & 0.82 & (0.73 to 0.93$)$ \\
\hline Dyslipidaemia, n (\%) & $1035(41.6)$ & $1745(46.2)$ & 0.80 & (0.72 to 0.88$)$ \\
\hline Chronic renal insufficiency, $\mathrm{n}(\%)$ & $321(12.9)$ & $521(13.8)$ & 0.73 & (0.63 to 0.85$)$ \\
\hline Chronic hepatic disease, $\mathrm{n}(\%)$ & $53(2.1)$ & $72(1.9)$ & 1.00 & (0.70 to 1.45$)$ \\
\hline Hyperthyroidism, n (\%) & $130(5.2)$ & $137(3.6)$ & 1.50 & (1.17 to 1.93$)$ \\
\hline Chronic obstructive pulmonary disease, $\mathrm{n}(\%)$ & $252(10.0)$ & $475(12.4)$ & 0.68 & $(0.57$ to 0.80$)$ \\
\hline Major gastrointestinal/cerebrovascular/ other bleeding events, n (\%) & $98(3.9)$ & $168(4.4)$ & 0.80 & (0.62 to 1.04$)$ \\
\hline $\begin{array}{l}\text { Prevalent cardiovascular disease } \\
\text { (CHD, peripheral arterial disease, myocardial infarction), } \mathrm{n}(\%)\end{array}$ & $467(18.9)$ & $1194(31.7)$ & 0.41 & $(0.36$ to 0.47$)$ \\
\hline Stent insertion, $\mathrm{n}(\%)$ & $140(5.6)$ & $527(13.9)$ & 0.33 & $(0.27$ to 0.40$)$ \\
\hline Heart valve dysfunction, $\mathrm{n}(\%)$ & $1106(44.2)$ & $1375(36.1)$ & 1.25 & (1.12 to 1.39$)$ \\
\hline Variables & Women $(n=2546)$ & Men $(n=3866)$ & Women-to-men $\mathrm{OR}^{*}$ & $95 \% \mathrm{Cl}$ \\
\hline Heart valve replacement, $\mathrm{n}(\%)$ & $150(6.0)$ & $201(5.3)$ & 1.09 & (0.88 to 1.37$)$ \\
\hline Heart failure, $n(\%)$ & $653(26.5)$ & 1150 (30.6) & 0.72 & (0.64 to 0.80$)$ \\
\hline Previous ischaemic stroke/TIA/other ischaemic thromboembolic event, $n$ (\%) & $406(16.2)$ & $555(14.5)$ & 1.04 & (0.90 to 1.20$)$ \\
\hline Sinus rhythm at baseline, $\mathrm{n}(\%)$ & $823(32.5)$ & $1134(29.5)$ & 1.37 & (1.22 to 1.53$)$ \\
\hline Adequate heart rate control (60-100 bpm) at baseline, $n(\%)$ & $1077(51.7)$ & $1605(51.0)$ & 0.98 & (0.87 to 1.09$)$ \\
\hline \multicolumn{5}{|l|}{ Medication } \\
\hline Antiplatelet agents, non-steroidal anti-inflammatory drugs, $\neq \mathrm{n}(\%)$ & $572(22.6)$ & $978(25.5)$ & 0.82 & $(0.73$ to 0.93$)$ \\
\hline Antiarrhythmic drugs, $\mathrm{n}(\%)$ & $1594(62.6)$ & $2257(58.4)$ & 1.31 & (1.18 to 1.46$)$ \\
\hline
\end{tabular}

Mean and SD and number and percentages are presented.

*Univariate ORs for gender were obtained by logistic regression adjusted for age and country.

tCHA $\mathrm{DS}_{2}-\mathrm{VASc}$ score included the extra point for female gender.

¥Medication as used in the HAS-BLED score. Lack of guideline compliance indicates lack of treatment with oral anticoagulant in the previous 12 months despite guideline indication without contraindication.

$A F$, atrial fibrillation; $\mathrm{CHA}_{2} \mathrm{DS}$ - $-\mathrm{VASC}$, congestive heart failure, hypertension, age $\geq 75$ years, diabetes mellitus, stroke, vascular disease, age 65-74 years, sex category; CHD, coronary heart disease; EHRA, European Heart Rhythm Association; HAS-BLED, hypertension, abnormal renal/liver function, stroke, bleeding history or predisposition, labile international normalised ratio, elderly, drugs/alcohol concomitantly; PREFER, PREvention of thromboembolic events - European Registry; TIA, transient ischaemic attack.

point estimates for these ORs were similar at 1-year follow-up, although CIs were wider due to the smaller number of procedures. Treatment varied by country, but the proportion of women and men receiving a specific therapy was comparable across European countries (data not shown).

\section{Incidence of major outcomes}

There was no evidence that men and women differed in the two outcomes: stroke/TIA/arterial thromboembolic events and major bleeding events (OR 1.08, 95\% CI (0.76 to 1.53$)$ and OR 0.91 , (0.67 to 1.24$)$, respectively) (figure 3 ). Women had lower age-adjusted and country-adjusted odds of coronary revascularisation (OR $0.35,95 \% \mathrm{CI}(0.22$ to 0.56$)$ ), lower odds of acute coronary syndrome (OR $0.60,(0.38$ to 0.93$)$ ), and lower odds of heart failure (OR $0.80,(0.68$ to 0.96$))$.

\section{One-year predictive models of major outcomes}

The stepwise regression results for each outcome are presented in online supplementary tables 4-8. Overall, there was no evidence that the strength of association between selected predictors and outcomes differed markedly between genders.

The predictive ability (AUC index) of our models performed similar to the established $\mathrm{CHA}_{2} \mathrm{DS}_{2}-$ VASc and HAS-BLED scores for stroke/TIA/arterial thromboembolic events and major bleeding (online supplementary table 9) in both genders. Overall, the predictive abilities of all models were moderate (AUC range: $0.58-0.69)$.

\section{DISCUSSION}

\section{Main Findings}

In a prospective European registry of patients with AF, women presented with different clinical baseline characteristics including disease history, AF symptoms, therapies to restore sinus rhythm and 1-year incidence of major cardiovascular outcomes. Women were older on average and showed a lower risk of acute coronary syndrome, coronary revascularisation events and development of heart failure compared with men over the 1-year follow-up. 

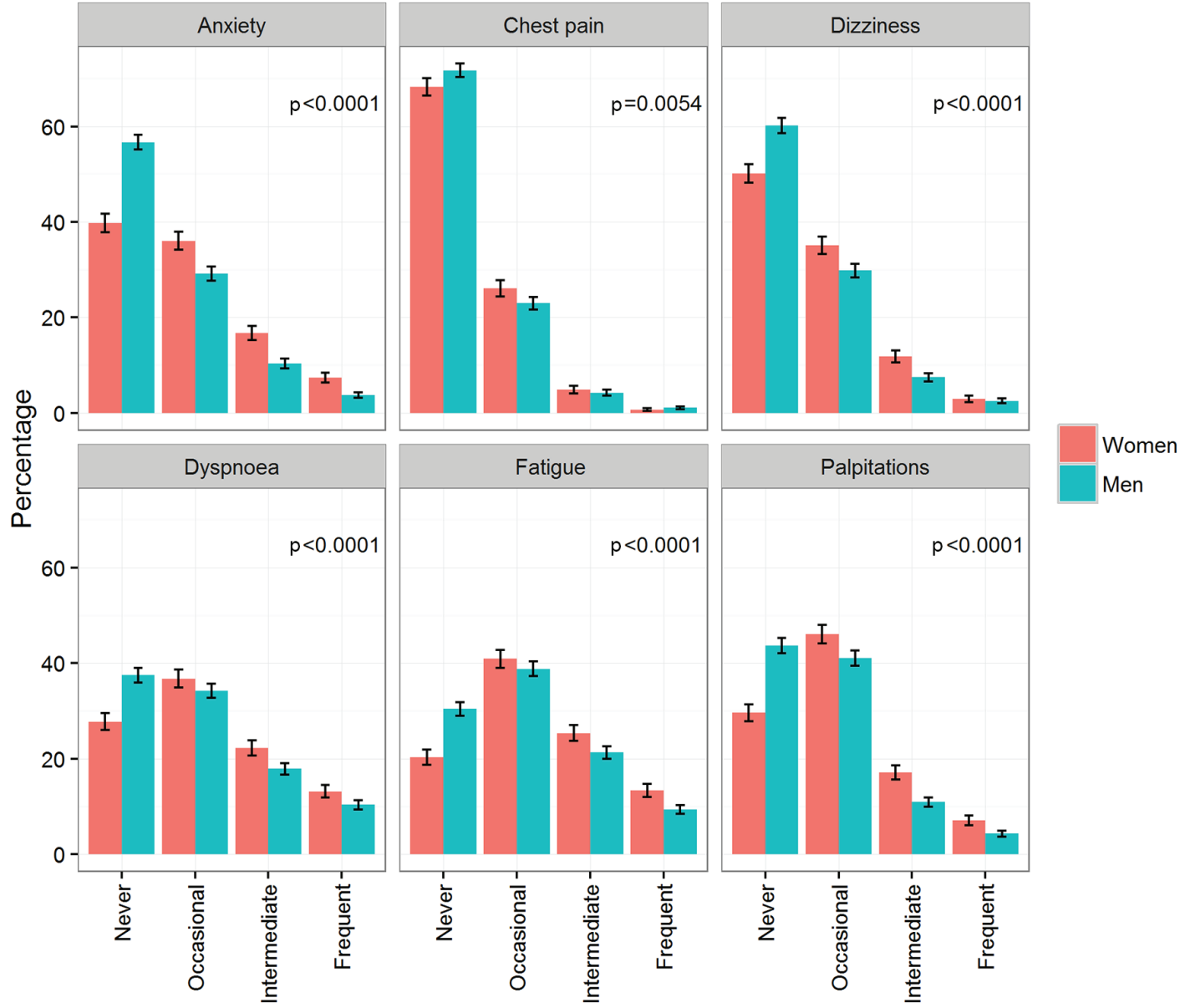

Figure 1 Symptoms according to the European Heart Rhythm Association classification at baseline by gender. Percentages and $95 \% \mathrm{Cl}$ are provided. $\mathrm{p}$ Values are derived from Freeman-Halton's extension of Fisher's exact test.
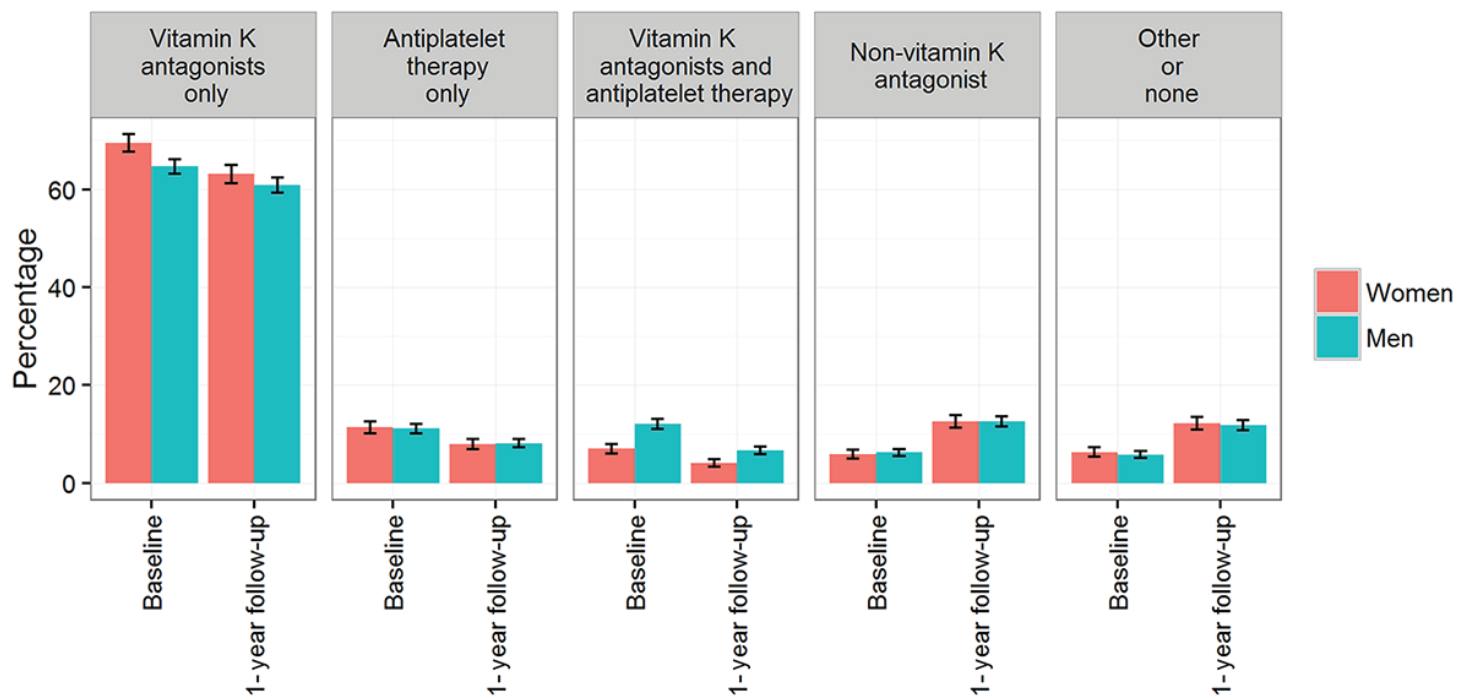

Figure 2 Prevalence of anticoagulation therapy in patients with atrial fibrillation at baseline and after 1-year follow-up by gender. Percentages and 95\% $\mathrm{Cl}$ are provided. $p$ Values are derived from Freeman-Halton's extension of Fisher's exact test; baseline, $p<0.0001$; follow-up, $p=0.001$.

There was no evidence of gender differences for arterial thromboembolic events or bleeding. We report possible differences between gender in the strength of association of clinical predictors and outcomes, suggesting that a gender-specific risk assessment and intervention strategies could be of advantage.

\section{Atrial fibrillation symptoms}

Our findings that women with AF reported more symptoms than men are in line with recent reports that fairly consistently described a higher subjective symptom burden in women. ${ }^{14-16}$ Truly asymptomatic AF, that is, no symptom history or current 
Table 2 ORs for gender for baseline prevalence and 1-year incidence for treatments to restore sinus rhythm

\begin{tabular}{|c|c|c|c|c|c|c|c|c|c|c|c|c|}
\hline \multirow[b]{3}{*}{ Treatment } & \multicolumn{6}{|c|}{ Baseline } & \multicolumn{6}{|c|}{ One-Year follow-up } \\
\hline & \multicolumn{2}{|c|}{ Women } & \multicolumn{2}{|c|}{ Men } & \multicolumn{2}{|c|}{ Women vs men } & \multicolumn{2}{|c|}{ Women } & \multicolumn{2}{|l|}{ Men } & \multicolumn{2}{|c|}{ Women vs men } \\
\hline & $\mathrm{N}$ & $\%$ & $\mathrm{~N}$ & $\%$ & OR & $95 \% \mathrm{Cl}$ & $\mathrm{N}$ & $\%$ & $\mathbf{N}$ & $\%$ & $\mathrm{OR}$ & $95 \% \mathrm{Cl}$ \\
\hline Pharmacological cardioversion & 511 & 20.2 & 716 & 18.6 & 1.24 & (1.08 to 1.41$)$ & 132 & 5.4 & 180 & 4.8 & 1.25 & (0.99 to 1.59 \\
\hline Electrical cardioversion & 379 & 14.9 & 795 & 20.6 & 0.78 & (0.68 to 0.90$)$ & 144 & 5.9 & 329 & 8.8 & 0.82 & (0.67 to 1.01 \\
\hline Ablation (pulmonary vein isolation) & 84 & 3.3 & 243 & 6.3 & 0.72 & (0.56 to 0.94$)$ & 68 & 2.8 & 170 & 4.5 & 0.88 & (0.66 to 1.19 \\
\hline Surgical therapy for atrial fibrillation & 10 & 0.4 & 36 & 0.9 & 0.45 & (0.22 to 0.93$)$ & 6 & 0.2 & 19 & 0.5 & 0.52 & $(0.20$ to 1.31 \\
\hline
\end{tabular}

ORs are age and country adjusted.

symptoms, was associated with the male gender in a prior observational registry study. ${ }^{17}$ Whether differences in illness perception and coping strategies or different major drivers causing AF underlie the lower symptom prevalence in men warrants further study.

\section{Therapies to restore sinus rhythm}

Despite more AF symptoms in women, men received more rhythm control interventions at baseline and also during the 1-year follow-up; for example, both the prevalence and 1-year incidence of electrical cardioversion, pulmonary vein isolation or surgical therapy for AF were lower in women, whereas antiarrhythmic drug use was slightly higher. Recent publications show a similar pattern. ${ }^{16}$ The reason why women with AF were less likely to receive non-pharmacological interventions to maintain sinus rhythm remains unclear. Besides symptom burden, clinical factors such as the presence of heart disease and other comorbidities and patient preferences enter the decision process for catheter-based ablation therapy. ${ }^{18}$

In our cohort, women presented more often with valvular dysfunction but otherwise presented fewer risk factors, except for age, which may explain the observed more conservative treatment pattern. Data on other cardiovascular interventions suggest that women undergo a less aggressive treatment than men. In non-ST elevation myocardial infarction, women were treated invasively less frequently despite higher risk of adverse events ${ }^{19}$ or received cardiac devices (eg, cardiac resynchronisation therapy), less often than men. ${ }^{20}$ Current evidence suggests that $\mathrm{AF}$ treatments are as effective in women as in men, ${ }^{21}$ supported by the current guideline recommendations that state that catheter or surgical ablation techniques should be regarded as equally effective in women and men. The success rates of sinus rhythm restoration appear to be similar in both genders. ${ }^{22}$ A possible explanation for the treatment differences may be patient choice. Women tend to refuse multiple ablation procedures even after initial success, which results in a lower overall maintenance of sinus rhythm due to fewer repeat procedures compared with men. ${ }^{22}$ To which extent physician preferences and recommendations play a role in treatment differences remains to be examined.

\section{Anticoagulation therapies}

As recommended in the guidelines for AF treatment, anticoagulation therapy was prescribed in the majority of patients, without any seeming difference between women or men. Similar results were reported in the Global Anticoagulant Registry in the Field-AF registry in $2014 .^{17}$ Thus, positive changes can be demonstrated compared with earlier studies, which showed that women were less likely to receive oral anticoagulants compared with men. ${ }^{23}$ The uptake of NOACs during the follow-up was also similar in both genders, with a doubling from about $6 \%$ at baseline to $12 \%$ after 1 -year follow-up. Considering evidence from a secondary analysis of NOAC trials ${ }^{24} 25$ and a recent meta-analysis, ${ }^{4}$ women appear to benefit from NOAC similarly to men. They may even have a higher net clinical benefit due to the high residual stroke risk in women treated with warfarin but lower stroke rates under NOAC therapy. Published data on the incidence of bleeding events are mostly from the warfarin era and have remained controversial regarding gender differences. ${ }^{26}$ Whether the absence of gender differences in bleeding risk in our patient cohort may be explained by the broader application of NOACs during the 1-year follow-up or relate to a genuine lack of gender differences should be studied in the future.

\section{Incidence of major outcomes}

A higher risk of stroke and other arterial thromboembolic events in women has been reported, most recently in a large meta-analysis, which however showed substantial heterogeneity among studies. ${ }^{2}$ Women in our study had $8 \%$ higher age-adjusted and country-adjusted odds of stroke/TIA/arterial thromboembolic events compared with men-an effect size much smaller than

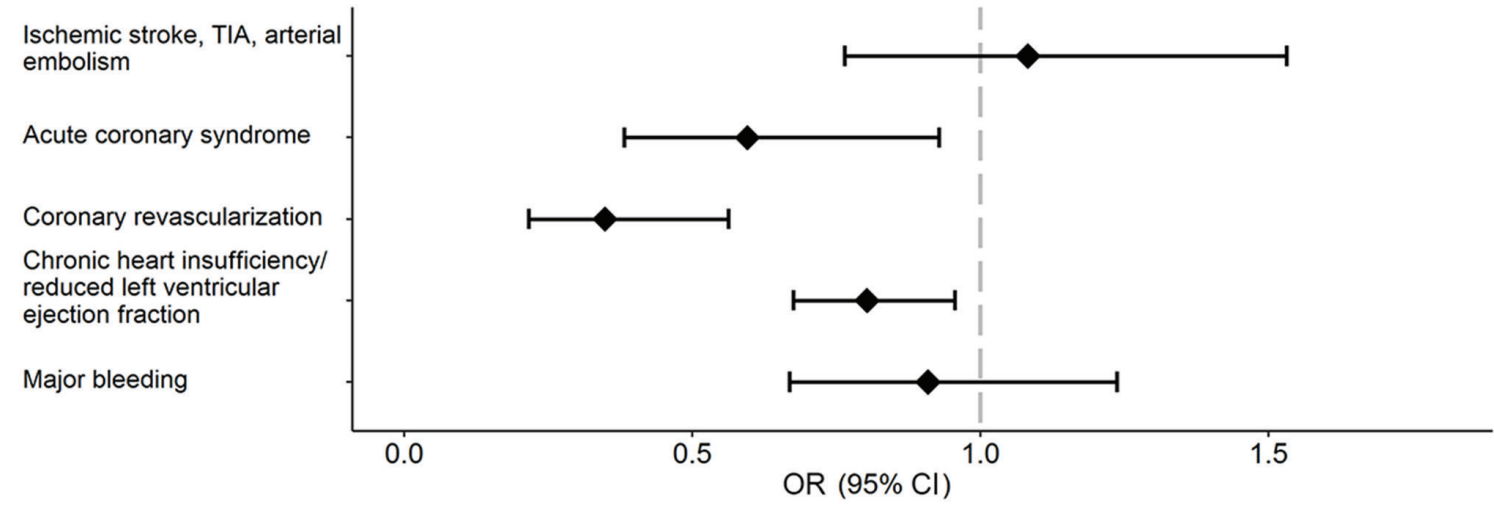

Figure 3 Age-adjusted and country-adjusted women-to-men ORs for 1-year major outcomes. An OR below 1 indicates a lower risk of developing the outcome in women. TIA, transient ischaemic attack. 
suggested by the meta-analysis. Due to the relatively short follow-up period of this study, the event numbers were small, and the uncertainty around our estimated effects were relatively large. At baseline, women accumulated a higher $\mathrm{CHA}_{2} \mathrm{DS}_{2}-\mathrm{VASc}$ score on average due to the extra point for female gender, which did not directly translate in a higher stroke risk during follow-up. Thus, the general addition of one point for gender, regardless of age, needs to be appraised carefully in practice. Women with a CHA,DS - VASc score of 1 are usually at a very low risk of stroke. ${ }^{27}$

Additionally, our results are derived from a cohort with a high proportion of adequate anticoagulation treatment. Underuse of anticoagulation in women despite relevant stroke risk has been suggested as another explanation for gender disparities in arterial thromboembolic outcomes. ${ }^{1526}$ The increased prescription of NOACs, with a possible more effective stroke risk reduction in women, may have contributed to smaller gender differences in stroke incidence.

AF has been recognised as a risk factor for myocardial infarction. ${ }^{628}$ Whether gender differences exist has remained controversial. Whereas earlier reports did not show differences in myocardial infarction incidence, ${ }^{26}{ }^{29}$ a recent population-based study observed a stronger association between AF and myocardial infarction in women. ${ }^{30}$ Our current data, which included the whole spectrum of acute coronary syndromes, indicate that women were at a lower short-term risk than men. Similarly, the outcome of coronary revascularisation was more often seen in men than in women.

Heart failure is an important complication in individuals with AF. In the current analyses, we observed a higher incidence of heart failure in men over the 1-year follow-up period. We have previously described possible gender differences in the susceptibility to heart failure in AF when examining subtypes of heart failure, that is, heart failure with preserved and reduced ejection fraction. ${ }^{5}$ Men had a higher incidence of heart failure with reduced ejection fraction, and women tended to have a higher risk of developing heart failure with preserved ejection fraction.

Clinical predictors of major outcomes revealed similar predictive ability in both genders, with overall moderate discriminatory ability. We observed possible differences in the strength of association in men compared with women. If validated, such differences may help estimate the risk factor burden and target AF treatment gender specifically. Despite being recently used to assess risk across diverse outcomes in patients with $\mathrm{AF}$, the $\mathrm{CHA}_{2} \mathrm{DS}_{2}-\mathrm{VASc}$ and HAS-BLED scores performed similarly to our predictive models.

\section{Limitations}

As common in registries, the accuracy and completeness of data may be limited, despite a high degree of standardisation and training in data acquisition across enrolling centres, which may lead to bias. The results were obtained in individuals that sought medical attention due to the rhythm disorder and may thus not be generalisable to all patients with AF. Over the 1-year time span of the registry, the number of serious medical outcomes was also limited compared with the number of predictor variables examined. The PREFER in AF registry enrolled patients in seven Western European countries. Therefore, patient characteristics, AF management and incidence of adverse events may not be generalisable to all European countries and other regions of the world. Such possible differences have been shown within the registry and are likely to be more pronounced for a comparison with other countries. ${ }^{8}$ Whereas differences in treatment

\section{Key messages}

What is already known on this subject?

There are significant gender differences in the epidemiology of atrial fibrillation. Data on clinical presentation, atrial fibrillationspecific interventions, and treatment and outcomes in a contemporary European cohort are limited.

\section{What might this study add?}

Women were more symptomatic ( $95.4 \%$ vs $89.8 \%$ in men). Prescription of oral anticoagulation therapy was comparable. Men were more aggressively treated (electrical cardioversion $20.6 \%$ vs $14.9 \%$; ablation $6.3 \%$ vs $3.3 \%$ ). Women had $40 \%$ lower odds of developing acute coronary syndrome and $20 \%$ lower odds of heart failure.

How might this impact on clinical practice? Gender-specific treatment of atrial fibrillation and prevention of adverse events need to be explored to improve atrial fibrillation care and outcomes.

choice were observed across countries in our cohort, the relative proportions of women and men undergoing a specific treatment were comparable.

In summary, we demonstrated gender differences in the clinical presentation, treatment and major clinical outcomes in a large, contemporary cohort, which call for gender-individualised research and care. Guideline-recommended anticoagulation medication for AF was prescribed in both men and women, with a good uptake of NOACs in both genders. Women were less likely to receive non-pharmacological therapies to restore sinus rhythm despite being more symptomatic. The observed gender disparities require detailed pathophysiological and clinical workup and may provide the opportunity to develop gender-specific preventive and therapeutic strategies for a disease that is reaching epidemic proportions worldwide.

\section{Author affiliations}

${ }^{1}$ Department of Cardiology, University Heart Center Hamburg-Eppendorf, German Center for Cardiovascular Research (DZHK) partner site Hamburg/Kiel/Lübeck,

Hamburg, Germany

${ }^{2}$ Medical Facility Pilsen of Charles University, Pilsen, Czech Republic

${ }^{3}$ Daiichi Sankyo Europe GmbH, Munich, Germany

${ }^{4}$ Department of Cardiology, Angiology, Nephrology and Conservative Intensive Care Medicine, Vivantes Klinikum Neukolln, Berlin, Germany

${ }^{5}$ School of Clinical \& Experimental Medicine, University of Birmingham, Birmingham, UK

${ }^{6} \mathrm{G}$. d'Annunzio University of Chieti-Pescara, Chieti, Italy

${ }^{7}$ Fondazione G. Monasterio, Pisa, Italy

${ }^{8}$ Institute of Cardiovascular Sciences, University of Birmingham and SWBH and UHB NHS Trust, Birmingham, UK

${ }^{9}$ AFNET, Münster, Germany

Acknowledgements This analysis of the PREFER in AF registry was initiated by the Thrombosis Exchange Meeting in AF, TEAM in AF, funded and sponsored by Daiichi Sankyo Europe. We thank all the participants for their time and efforts with the establishment of the registry.

Contributors RBS designed the analysis, interpreted the data and wrote the manuscript. LP designed the analysis, performed the statistical analysis and critically reviewed and revised the manuscript. FMO interpreted the data, wrote parts of the manuscript and critically reviewed the manuscript. ML designed the study, obtained the funding and critically reviewed the manuscript. NR wrote parts of the manuscript, interpreted the data and critically reviewed the manuscript. SB critically reviewed the manuscript. HD critically reviewed the manuscript. DK interpreted the data and critically revised the manuscript. RDC designed the study, obtained the funding and critically reviewed the manuscript. PK interpreted the data, designed the study, obtained the funding and critically reviewed the manuscript. 
Funding This project has received funding from the European Research Council under the European Union's Horizon 2020 Research and Innovation Programme (grant agreement number 648131). This work was performed in the context of the Junior Research Alliance symAtrial project funded by the German Ministry of Research and Education (BMBF 01ZX1408A) e:Med - Systems Medicine program (RBS). RBS is funded by Deutsche Forschungsgemeinschaft (German Research Foundation) Emmy Noether Program SCHN 1149/3-1 (RBS). The PREFER in AF registry has been funded by Daiichi Sankyo Europe.

Competing interests The PREFER in AF study sponsor via a contract research organisation (SSS International Clinical Research GmbH, Munich, Germany) was Daiichi Sankyo Europe GmbH, Munich. The study has an independent scientific steering committee. RDC reports that his institution received research grant support from Boehringer-Ingelheim, Bayer, Bristol-Myers Squibb/Pfizer and Roche and honoraria for lectures and/or consulting from Boehringer-Ingelheim, Bayer and Bristol-Myers Squibb/Pfizer, Daiichi Sankyo, Lilly, AstraZeneca, Merck and Novartis. PK receives further research support from the European Union (grant agreement number 633193 (CATCH ME)), British Heart Foundation (FS/13/43/30324), Medical Research Council (UK), Leducq Foundation, German Centre for Heart Research and several drug and device companies active in atrial fibrillation and has received honoraria from several such companies. He is listed as an inventor on two pending patents (WO 2015140571 and WO 2016012783) filed by the University of Birmingham. DK reports grants from Menarini outside the submitted work but during the conduct of the study and professional development support from Daiichi Sankyo.

Ethics approval Approvals were obtained from leading and local ethics committees as required by national regulations in Austria, Germany, Switzerland, Italy, Spain and the UK before the start of enrolment at the sites. For France, no specific approval process was applicable for non-interventional studies.

Provenance and peer review Not commissioned; externally peer reviewed.

Open Access This is an Open Access article distributed in accordance with the Creative Commons Attribution Non Commercial (CC BY-NC 4.0) license, which permits others to distribute, remix, adapt, build upon this work non-commercially, and license their derivative works on different terms, provided the original work is properly cited and the use is non-commercial. See: http://creativecommons.org/ licenses/by-nc/4.0/

(c) Article author(s) (or their employer(s) unless otherwise stated in the text of the article) 2017. All rights reserved. No commercial use is permitted unless otherwise expressly granted.

\section{REFERENCES}

1 Chugh SS, Havmoeller R, Narayanan K, et al. Worldwide epidemiology of atrial fibrillation: a Global Burden of Disease 2010 Study. Circulation 2014;129:837-47.

2 Emdin CA, Wong CX, Hsiao AJ, et al. Atrial fibrillation as risk factor for cardiovascular disease and death in women compared with men: systematic review and metaanalysis of cohort studies. BMJ 2016;532:h7013.

3 Lip GY, Nieuwlaat R, Pisters R, et al. Refining clinical risk stratification for predicting stroke and thromboembolism in atrial fibrillation using a novel risk factor-based approach: the euro heart survey on atrial fibrillation. Chest 2010;137:263-72.

4 Pancholy SB, Sharma PS, Pancholy DS, et al. Meta-analysis of gender differences in residual stroke risk and major bleeding in patients with nonvalvular atrial fibrillation treated with oral anticoagulants. Am J Cardio/ 2014:113:485-90.

5 Schnabel RB, Rienstra M, Sullivan LM, et al. Risk assessment for incident heart failure in individuals with atrial fibrillation. Eur J Heart Fail 2013:15:843-9.

6 Soliman EZ, Lopez F, O'Neal WT, et al. Atrial Fibrillation and risk of ST-segmentelevation versus non-ST-segment-slevation myocardial infarction: the Atherosclerosis risk in communities (ARIC) study. Circulation 2015;131:1843-50.

7 Kirchhof P, Benussi S, Kotecha D, et al. ESC Guidelines for the management of atrial fibrillation developed in collaboration with EACTS: The task force for the management of atrial fibrillation of the European Society of Cardiology (ESC) Developed with the special contribution of the European Heart Rhythm Association (EHRA) of the ESCEndorsed by the European Stroke Organisation (ESO). Eur Heart J 2016;50:e1-e88.

8 Kirchhof $\mathrm{P}$, Ammentorp B, Darius $\mathrm{H}$, et al. Management of atrial fibrillation in seven European countries after the publication of the 2010 ESC Guidelines on atrial fibrillation: primary results of the PREvention of thromboemolic events--European Registry in Atrial Fibrillation (PREFER in AF). Europace 2014;16:6-14.
9 Camm AJ, Kirchhof P, Lip GY, et al. European Heart Rhythm Association. European Association for Cardio-Thoracic Surgery. Guidelines for the management of atrial fibrillation: the task force for the management of atrial fibrillation of the European Society of Cardiology (ESC). Eur Heart J 2010;31:2369-429.

10 Kirchhof $\mathrm{P}$, Auricchio A, Bax J, et al. Outcome parameters for trials in atrial fibrillation: recommendations from a consensus conference organized by the German Atrial Fibrillation Competence NETwork and the European Heart Rhythm Association. Europace 2007:9:1006-23.

11 Lip GY. Implications of the CHA(2)DS(2)-VASC and HAS-BLED Scores for thromboprophylaxis in atrial fibrillation. Am J Med 2011;124:111-4.

12 R Core Team. R: A Language and Environment for Statistical Computing. In: R Foundation for Statistical Computing. Vienna, Austria: 2016.

13 Wickham H. ggplot2. Elegant Graphics for Data Analysis. New York: Springer-Verlag, 2009.

14 Potpara TS, Marinkovic JM, Polovina MM, et al. Gender-related differences in presentation, treatment and long-term outcome in patients with first-diagnosed atrial fibrillation and structurally normal heart: the Belgrade atrial fibrillation study. Int J Cardiol 2012;161:39-44

15 Dagres N, Nieuwlaat R, Vardas PE, et al. Gender-related differences in presentation, treatment, and outcome of patients with atrial fibrillation in Europe: a report from the Euro Heart Survey on Atrial Fibrillation. J Am Coll Cardiol 2007;49:572-7.

16 Lip GY, Laroche C, Boriani G, et al. Sex-related differences in presentation, treatment, and outcome of patients with atrial fibrillation in Europe: a report from the Euro Observational Research Programme Pilot survey on Atrial Fibrillation. Europace 2015;17:24-31.

17 Boriani G, Laroche C, Diemberger I, et al. Asymptomatic atrial fibrillation: clinical correlates, management, and outcomes in the EORP-AF Pilot General Registry. Am J Med 2015;128:509-18.

18 Calkins H, Kuck KH, Cappato R, et al. 2012 HRS/EHRA/ECAS expert consensus statement on catheter and surgical ablation of atrial fibrillation: recommendations for patient selection, procedural techniques, patient management and follow-up, definitions, endpoints, and research trial design. Heart Rhythm 2012;9:632-96.

19 Blomkalns AL, Chen AY, Hochman JS, et al. CRUSADE Investigators. CRUSADE Investigators. Gender disparities in the diagnosis and treatment of non-ST-segment elevation acute coronary syndromes: large-scale observations from the CRUSADE (Can Rapid Risk Stratification of Unstable Angina Patients Suppress Adverse Outcomes With Early Implementation). J Am Coll Cardio/ 2005;45:832-7.

20 Sridhar AR, Yarlagadda V, Parasa S, et al. Cardiac Resynchronization Therapy: US Trends and Disparities in Utilization and Outcomes. Circ Arrhythm Electrophysiol 2016:9:e003108

21 Forleo GB, Tondo C, De Luca L, et al. Gender-related differences in catheter ablation of atrial fibrillation. Europace 2007:9:613-20.

22 Winkle RA, Mead RH, Engel G, et al. Long-term results of atrial fibrillation ablation: the importance of all initial ablation failures undergoing a repeat ablation. Am Heart J 2011;162:193-200.

23 DeWilde S, Carey IM, Emmas C, et al. Trends in the prevalence of diagnosed atrial fibrillation, its treatment with anticoagulation and predictors of such treatment in UK primary care. Heart 2006;92:1064-70.

24 Vinereanu D, Stevens SR, Alexander JH, et al. Clinical outcomes in patients with atrial fibrillation according to sex during anticoagulation with apixaban or warfarin: a secondary analysis of a randomized controlled trial. Eur Heart J 2015;36:3268-75.

25 Avgil Tsadok M, Jackevicius CA, Rahme E, et al. Sex differences in dabigatran use, safety, and effectiveness in a population-based cohort of patients with atrial fibrillation. Circ Cardiovasc Qual Outcomes 2015;8:593-9.

26 Humphries KH, Kerr CR, Connolly SJ, et al. New-onset atrial fibrillation: sex differences in presentation, treatment, and outcome. Circulation 2001;103:2365-70.

27 Friberg L, Skeppholm M, Terént A. Benefit of anticoagulation unlikely in patients with atrial fibrillation and a CHA2DS2-VASc score of 1. J Am Coll Cardiol 2015;65:225-32.

28 Soliman EZ, Safford MM, Muntner P, et al. Atrial fibrillation and the risk of myocardial infarction. JAMA Intern Med 2014;174:107-14.

29 Chao TF, Huang YC, Liu CJ, et al. Acute myocardial infarction in patients with atrial fibrillation with a CHA2DS2-VASC score of 0 or 1: a nationwide cohort study. Heart Rhythm 2014;11:1941-7.

30 Soliman EZ, Safford MM, Muntner P, et al. Atrial fibrillation and the risk of myocardial infarction. JAMA Intern Med 2014;174:107-14. 